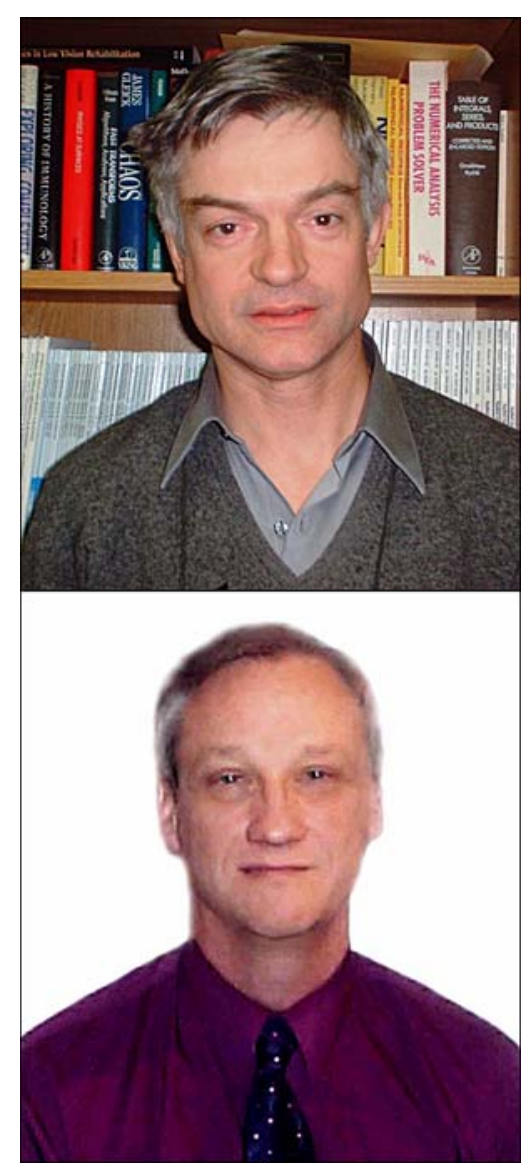

Gislin Dagnelie, PhD; Ronald A. Schuchard, PhD

\section{The state of visual prosthetics-Hype or promise?}

The miracle of restoring sight to the blind, told in legends and myths throughout human cultures and history, is moving from dream to possibility in our times. Several dozen groups around the world now claim that electronic or neurochemical prostheses may soon restore some measure of functional vision. This editorial examines such claims by reviewing the proposed approaches and assessing their promise. We base our opinions on our involvement with some of these efforts and on presentations and discussions during the most recent biennial Eye and the Chip: World Congress on Artificial Vision organized by the Detroit Institute of Ophthalmology in June 2006; for a recent overview of many current activities in this area, see Dagnelie [1].

The retina extensively preprocesses and reduces image information, and the cortical map and magnification add further complexity; therefore, functional replacement of neural elements should be done at the earliest available stage of visual processing to limit "surrogate" processing that provides but a poor substitute for the native system. Thus, for example, a retinal prosthesis should be used if the nerve fibers and optic nerve are intact, such that the prosthesis stimulates the most distal remaining cell population available. Despite such considerations, the first attempts at vision restoration almost 40 years ago were directed at the visual cortex [2]. Early cortical implants used electrodes placed between the meninges, avoiding the complications of glial cell encapsulation plaguing most penetrating electrodes. A mapping procedure is used to determine the location of each electrode's phosphene in the visual field, and real-time image processing and stimulation software then extracts and projects contours onto the best-matching electrodes. These phosphenes do not convey real-form vision, yet they do allow the wearer crude localization of outlines in the scene. Newer penetrating probes such as the 100-electrode array developed at the University of Utah-currently being considered for use by the European CortiVis consortium - and the "hatpin" electrodes-developed at the National Institute of Neurological Disorders and Stroke and since adopted by the group at the Illinois Institute of Technology - may provide smaller phosphenes, and thus better resolution, at much lower and safer charge injection levels.

In the early 1990s, intraoperative stimulation in patients with late-stage retinitis pigmentosa (RP) showed that phosphenes could be elicited from blind retinal areas long after photoreceptor degeneration [3]. Researchers are working on various implants in which a matrix of electrodes stimulates the surviving retinal cells with imagery collected by an external camera and reduced to match the resolution of the electrode array. Two research groups have progressed to the stage of prototype implants in patients with late-stage RP. A consortium formed by Second Sight Medical Products LLC and by the Doheny Retina Institute has performed six implants of $4 \times 4$ electrode arrays 
based on cochlear implant electronics; these patients can detect phosphenes at individual electrodes, discriminate crude shapes upon multiple electrode stimulation, and recognize simple stimuli presented via a head-mounted camera. A consortium formed by IIP Technologies GmbH and German universities has performed several 50-electrode implants and is reporting that patients have similar discrimination abilities with computer-generated stimuli.

Other retinal prosthesis groups are exploring integrated concepts in which the eye's optics become part of a prosthetic imaging device, which in turn generates the signals to drive the secondary neurons. The simplest implementation of such a device, an array of small photodiodes under the retina, appears to have a neurotrophic beneficial effect in RP patients with some remaining vision; 30 patients have received this Optobionics ASR implant in Food and Drug Administration trials [4]. The addition of signal amplification and pulse modulation in a multilayered subretinal chip, which is a more complex approach, is being implemented by a German consortium formed by Retina Implant AG, and has recently been implanted for periods up to 2 months. A very different approach to such an integrated system is the conversion of secondary retinal neurons into photoreceptors by (genetically or otherwise) inserting photosensitive molecules found in plants, such as Photosystem II complex; however, bringing such techniques to the animal or human retina is likely to take many years.

Only one group thus far has pursued the concept of a prosthesis that stimulates the optic nerve, presumably because of the difficulty of creating an orderly map of the visual world in this high-density structure. Two patients who have had cuff electrodes implanted around the optic nerve can discriminate a very limited set of stimuli from an external camera; higher resolution is unlikely to be achieved without a radical change in electrode technology.

One may be tempted to view visual prosthesis development as a long yet straightforward problem of bioengineering in which the eventual success is contingent only on development of the right methods and materials and, ultimately, on time and monetary investment. Such an impression would be highly simplistic, however. Fundamental restrictions severely limit the potential for functional visual prostheses at the present time, which may or may not be overcome with present technology.

The first and perhaps most vexing problem is that remaining neurons in the degenerated retina do not retain their normal properties following photoreceptor degeneration. Recent microanatomical studies provide evidence for extensive reorganization of the connections among surviving retinal neurons and for cell migration outside their customary layers. Cells being stimulated by a retinal prosthesis may pass these signals to multiple cell types and functional streams that would not be interconnected in the normal retina, and signals may propagate over substantial distances. On the other hand, the adaptive response of retinal tissue may allow innovative interfacing between implants and retinal cells. For example, experiments at Stanford University have shown that certain retinal neurons tend to migrate into cavities between stimulating electrodes. Similarly, tissue culture studies from several laboratories (Stanford University, Tokyo Institute of Technology, Wayne State University) have demonstrated that targeted neurotransmitter release by microfluidic assemblies will cause some cell types to form close contacts with these devices.

A second limiting factor in signal transmission from electrodes to neural tissue is electrode geometry. Surface electrodes used in both cortical and retinal implants are located at distances up to $100 \mu \mathrm{m}$ from the target cells, prompting a need for large stimulus amplitudes and hence large surface areas to stay within safe current density limits and avoid electrochemical reactions at the electrode surface. Consequently, spatial resolution of these electrodes is limited to spacing on the order of $1^{\circ}$ in the visual field, i.e., 20/1200 visual acuity. Penetrating electrode technologies are being developed for intracortical applications, but a careful trade-off between electrode size, current density, and mechanical tissue damage will be required to find a compromise that allows higher resolution under safe long-term conditions.

A third factor concerning adaptation to any prosthesis with a camera outside the eye is the role of eye movements in visual tasks and the potentially 
difficult adjustment to their anomalous effects: eye movements will not cause the expected image shift and head-worn camera image movements will provide an inappropriate compensatory eye movement because of the vestibule-ocular reflex. A possible solution to this problem would be to equip visual prostheses with an eye tracker that can direct the image processor to execute the appropriate image shift. Based on observations in simulated prosthetic vision tests, however, prosthesis wearers likely will learn to reduce their eye movements and execute targeted head/camera movements to achieve the desired image shifts [5].

A final factor limiting the success of most visual prostheses is the unknown ability of patients with a long history of blindness to use the visual information provided by implanted electrode arrays. Patients without early visual experience are known to have extreme problems effectively using restored visual information. Even those who once had good vision may have great difficulty learning to function with the low resolution and limited extent provided by retinal implants or the irregular phosphene configuration provided by cortical implants. Early indications from implant wearers suggest that the plasticity of the visual system will allow a considerable degree of adaptation, so we may yet be surprised by implant wearers' adaptive abilities with even very early prosthetic devices.

Adaptations to visual prosthesis wearers' eye and head movements as well as visual perception should not be left to chance. Rather, we in the field of vision rehabilitation should recognize the field of visual prosthetics as a wide-open opportunity to contribute our expertise. While the tools may be new, the rehabilitation process itself is analogous to functional rehabilitation in every other field, and once we familiarize ourselves with the technology and understand its potential and limitations, we are in the best position to apply our professional training and skills to the rehabilitation of newly restored vision. For the best rehabilitation for visual prosthesis wearers, outcome measures need to be identified and possibly developed to provide evidence-based rehabilitation practice.
In summary, we believe that cautious optimism regarding the future of visual prostheses is reasonable. While the engineering challenges are considerable and the physiological and anatomical limitations real, crude levels of vision restoration through retinal and cortical implants are certainly on the horizon. We are encouraged that the Department of Veterans Affairs (VA) has joined the quest to develop functional visual prostheses by its sponsorship of both the Center for Innovative Visual Rehabilitation at the Boston VA Hospital, an expansion of the Boston retinal Implant Project [6] with collaborations at Massachusetts Eye and Ear Infirmary, Harvard Medical School, and Massachusetts Institute of Technology, and of prosthetic evaluation projects (human and animal) at the Atlanta VA Rehabilitation Center for Aging Veterans with Vision Loss.

On the other hand, one must realize that the coming years will only mark the dawn of visual prosthetics. Early implant recipients certainly will experience unforeseen difficulties in fully understanding the visual information provided by these implants and learning to use them to their advantage. This area is where we as rehabilitation specialists have a crucial role to play.

\section{REFERENCES}

1. Dagnelie G. Visual prosthetics 2006: Assessment and expectations. Expert Rev Med Devices. 2006;3(3): 315-25. [PMID: 16681453]

2. Dobelle WH, Mladejovsky MG, Girvin JP. Artificial vision for the blind: Electrical stimulation of visual cortex offers hope for a functional prosthesis. Science. 1974;183:440-44. [PMID: 4808973]

3. Humayun MS, De Juan E Jr, Dagnelie G, Greenberg RJ, Propst RH, Phillips DH. Visual perception elicited by electrical stimulation of retina in blind humans. Arch Ophthalmol. 1996;114(1):40-46. [PMID: 8540849]

4. Chow AY, Chow VY, Packo KH, Pollack JS, Peyman GA, Schuchard RA. The artificial silicon retina microchip for the treatment of vision loss from retinitis pigmentosa. Arch Ophthalmol. 2004;122(4):460-69.

[PMID: 15078662] 
JRRD, Volume 44, Number 3, 2007

5. Dagnelie G, Walter M, Yang L. Playing checkers: Detection and eye-hand coordination in simulated prosthetic vision. J Mod Opt. 2006;53(9):1325-42.

6. Rizzo JF 3rd, Wyatt J, Loewenstein J, Kelly S, Shire D. Methods and perceptual thresholds for short-term electrical stimulation of human retina with microelectrode arrays. Invest Ophthalmol Vis Sci. 2003;44(12): 5355-61. [PMID: 14638738]
Gislin Dagnelie, $P h D^{1}$; Ronald A. Schuchard, $P h D^{2 *}$

${ }^{1}$ Associate Professor of Ophthalmology, Johns Hopkins University School of Medicine, Baltimore, MD; ${ }^{2}$ Director, VA Rehabilitation Research and Development Center; Associate Professor of Neurology, Emory University School of Medicine, Decatur, GA

*Email: rschuch@emory.edu

DOI: 10.1682/JRRD.2006.08.0098 